\title{
A novel method to improve the spatial resolution of GEM neutron detectors with a stopping layer
}

This paper was downloaded from TechRxiv (https://www.techrxiv.org).

LICENSE

CC BY-NC-SA 4.0

SUBMISSION DATE / POSTED DATE

24-08-2021 / 26-08-2021

\section{CITATION}

Yang, Tao; Zhou, Jianrong; Zhou, Xiaojuan; Zhu, Lin; Zhu, Hangyu; Zhou, Jianjin; et al. (2021): A novel method to improve the spatial resolution of GEM neutron detectors with a stopping layer. TechRxiv. Preprint. https://doi.org/10.36227/techrxiv.16423977.v1

$\mathrm{DOI}$

10.36227/techrxiv.16423977.v1 


\title{
A novel method to improve the spatial resolution of GEM neutron detectors with a stopping layer
}

\author{
Tao Yang, Jianrong Zhou, Xiaojuan Zhou, Lin Zhu, Hangyu Zhu, Jianjin Zhou, Yuanguang \\ Xia, Yangdong Wei, Xingfen Jiang, Wenqin Yang, Gui'an Yang, Songlin Wang, Yuguang Xie, \\ Zhijia Sun, Qun Ouyang, Jingtao Zhu, and Yuanbo Chen. ${ }^{1}$
}

\begin{abstract}
The gas electron multiplier (GEM) detector in combination with a solid converter has been widely used in the field of neutron detection in recent decades. Typically, the sub-millimeter is an important indicator for neutron detectors to satisfy many applications requiring a high spatial resolution. This paper proposes a novel method to improve the spatial resolution of ceramic GEM detectors by adding a stopping layer on top of the solid ${ }^{10} \mathrm{~B}_{4} \mathrm{C}$ neutron converter. This will restrict the emission of the secondary ion products of large angles and consequently improve the spatial resolution. The Monte Carlo program FLUKA is used to validate the method, and the verification experiments are carried out at the beam line \#20 (BL20) of the China Spallation Neutron Source (CSNS). The experimental results are approximately in agreement with the simulations. The measured spatial resolution is $1.61 \mathrm{~mm}$ for the GEM neutron detector operated at ambient pressure with a 1- $\mu \mathrm{m}$-thick ${ }^{10} \mathrm{~B}_{4} \mathrm{C}$ converter, and it is improved to $\sim 0.8 \mathrm{~mm}$ by coating a 3 - $\mu \mathrm{m}$-thick titanium on top of the ${ }^{10} \mathrm{~B}_{4} \mathrm{C}$ converter.
\end{abstract}

Index Terms - gas electron multiplier (GEM), neutron detector, high spatial resolution, sub-millimeter, stopping layer, Monte Carlo simulation, neutron beam line.

\section{INTRODUCTION}

$\mathrm{T}$ HE CSNS, as the fourth high-flux pulsed spallation neutron source facility in the world, had generated the neutron beam by the spallation reaction of $1.6-\mathrm{GeV}$ protons striking on the tungsten target in August 2017, and now its beam power is 100 $\mathrm{kW}$ and repetition rate $25 \mathrm{~Hz}$. The CSNS accelerator is mainly comprised of a $50-\mathrm{keV} \mathrm{H}^{-}$ion source, a $3-\mathrm{MeV}$ radio frequency quadrupole (RFQ) accelerator, an $80-\mathrm{MeV}$ drift tube linac (DTL), and a 1.6-GeV proton rapid cycle synchrotron (RCS) [1]. CSNS has built the first three neutron instruments at present, which are the small-angle neutron scattering spectrometer (SANS), the multi-purpose reflectometer (MR), and the general-purpose powder diffractometer (GPPD), respectively. In phase II of CSNS, the beam power will be raised to $500 \mathrm{~kW}$ and the new seventeen neutron instruments will be built.

This work was supported by the National Key R\&D Program of China (Grant No. 2017YFA0403702), the National Natural Science Foundation of China (Grant Nos. U1832119, 11635012 and 11775243), Youth Innovation Promotion Association CAS, China, and Guangdong Basic and Applied Basic Research Foundation, China (Grant No. 2019A1515110217). (Corresponding Authors: Jianrong Zhou, Zhijia Sun, Qun Ouyang).

Tao Yang, Jianrong Zhou, Xiaojuan Zhou, Lin Zhu, Jianjin Zhou, Yuanguang Xia, Yangdong Wei, Xingfen Jiang, Wenqin Yang, Gui'an Yang, Songlin Wang, Yuguang Xie, Zhijia Sun, Qun Ouyang, and Yuanbo Chen are
Neutron detectors are the essential components for neutron scattering instruments, among which ${ }^{3} \mathrm{He}$ detectors played a dominant role in the past for these instruments because of the high detection efficiency and the excellent $\mathrm{n} / \gamma$ discrimination ability [2]. However, for a decade or more, due to the ${ }^{3} \mathrm{He}$ shortage crisis since 2008, other ${ }^{3} \mathrm{He}$-alternative techniques have been extensively researched and developed in the neutron detection field $[3,4]$. The frequently-used alternative isotopes are ${ }^{10} \mathrm{~B}$ and ${ }^{7} \mathrm{Li}$, their cross sections for thermal neutrons (@0.025 eV) are respectively 3840 and 940 barns [5]. The natural abundance of ${ }^{10} \mathrm{~B}$ is $\sim 19.6 \%$ compared with that $\sim 7.4 \%$ for ${ }^{6} \mathrm{Li}$, so the enrichment of ${ }^{10} \mathrm{~B}$ is cost-effective. Technically speaking, it is not hard to produce ${ }^{10} \mathrm{~B}$-based converter films in reasonable sizes via evaporation or magnetron-sputtering techniques. Besides, the solid boron or $\mathrm{B}_{4} \mathrm{C}$ is a non-hazardous material, whose chemical properties are stable and therefore the solid ${ }^{10} \mathrm{~B}$-based detectors are environmentally safe.

The GEM detector as a type of micro-pattern gaseous detectors (MPGDs), was introduced by F. Sauli in 1997 [6]. Since then, the GEM-based detectors were widely used in particle physics and the field of position-sensitive radiation detection. GEM detectors have the advantages of high ratecapabilities, good detection efficiency, good timing and spatial resolution, radiation resistance, easy assembly, and the potential for large areas fabrication [7] By the combination of the neutron converters, GEM detectors can be applied in neutron detection. Many research works have been carried out for the GEM-based neutron detectors (nGEM) [8-19]. Klein et al. had developed the CASCADE detector system at Heidelberg University [12], they utilized the ${ }^{10} \mathrm{~B}$-coated GEM foils and stacked them to cumulate the detection efficiency (typically 6 layers); the spatial resolution was about $2.6 \mathrm{~mm}$ for the detector operated at ambient gas pressure; and the detection efficiency could reach 35\% for 1.8 A neutrons and 55\% for $5 \AA$ A neutrons, respectively. Ohshita et al. also researched on the GEM-based neutron detector and monitor at the Japan Proton Accelerator Research Complex (J-PARC) [13]; the ${ }^{10} \mathrm{~B}$ converter with a thickness of $0.02 \mu \mathrm{m}$ was coated on the cathode plate; the

with the State Key Laboratory of Particle Detection and Electronics, Institute of High Energy Physics, Chinese Academy of Sciences (CAS), Beijing 100049, China; Spallation Neutron Source Science Center, Dongguan 523803, China; and University of Chinese Academy of Sciences, Beijing 100049, China. (email: zhoujr@ihep.ac.cn, sunzj@ihep.ac.cn, ouyq@ihep.ac.cn).

Hangyu Zhu and Jingtao Zhu are with the MOE Key Laboratory of Advanced Micro-Structured Materials, School of Physics Science and Engineering, Tongji University, Shanghai 200092, China. 
spatial resolution was about $1.2 \mathrm{~mm}$ and detection efficiency of $0.0269 \%$ was obtained. Since 2013, the ceramic thick GEM (THGEM) has been developed for the thermal neutron detection at the CSNS [17-19]. The GEM foils fabrication adopts the printed circuit board (PCB) technology and the mechanical drilling method in a ceramic substrate, the foil is made of a $170-\mu \mathrm{m}$-thick ceramic substrate where both surfaces have a $15-\mu \mathrm{m}$-thick copper cladding. The neutron converter is coated on the cathode for the single GEM or also coated on the GEM foils for the stacked CASCADE-like GEM [17]. The single GEM had measured a detection efficiency of $\sim 4.2 \%$ for $1.59 \AA$ neutrons and a spatial resolution $\sim 2.7-3.0 \mathrm{~mm}$ with a strip pitch of $1.56 \mathrm{~mm}$. The stacked GEM had measured a detection efficiency of $\sim 54 \%$ for $4.9 \AA$ neutrons, and a spatial resolution $\sim 2.8-3.0 \mathrm{~mm}$.

The spatial resolution is one of the most parameters for determining the performance of a position-sensitive detector system. The spatial resolution of GEM-based neutron detectors with a solid neutron converter depends on the emitting angle of the secondary charged particles, the track length of charged particles in the working gas, the electron diffusion, and the pitch of the read-out strips [15]. A GEM neutron detector with a high spatial resolution (sub-millimeter) under a high flux of neutrons is requested for the future neutron scattering and reflectometry instruments, e.g., the planned energy-resolved neutron imaging instrument (ERNI) and the very small-angle neutron scattering spectrometer (VSANS) in the CSNS, etc. There exist several methods to improve the spatial resolution. One can increase the working-gas pressure, e.g., as indicated in [12], the spatial resolution of the CASCADE detector is improved from $2.6 \mathrm{~mm}$ at a pressure of 1 bar to $\sim 1.3 \mathrm{~mm}$ at 3.5 bar. Narrowing the strip pitch is also an effective means. For almost the same workinggas pressure, the GEM neutron detector at the J-PARC achieved a $1.2 \mathrm{~mm}$ resolution corresponding to a strip pitch of $0.8 \mathrm{~mm}$ $[11,13]$, which is better than $2.6 \mathrm{~mm}$ achieved by the CASCADE detector with a strip pitch of $1.56 \mathrm{~mm}$. However, the above methods have limitations to further improve the spatial resolution to the sub-millimeter level, and the best result is $1.2 \mathrm{~mm}$ achieved by the research group at the J-PARC. In recent years, a novel readout scheme based on the timeprojection-chamber (TPC) concept was used in the GEM neutron detectors [20-23] to improve the spatial resolution. The TPC-like method in these works identifies the start time of the ion tracks, which could reconstruct the original vertex of the ion tracks. It could almost eliminate the influence of the ion-track angle and significantly improve the spatial resolution of neutron GEM detectors to the sub-millimeter level, and spatial resolution about $0.25-0.6 \mathrm{~mm}$ was reported for thermal and fast GEM neutron detectors.

This paper proposes a novel method by coating a titanium layer as a stopping layer on top of the ${ }^{10} \mathrm{~B}_{4} \mathrm{C}$ layer to improve the spatial resolution of the solid-boron-converter based GEM neutron detectors. The stopping layer could constrain the emission of the secondary ions with large angles (implies a large transverse projection), and only allows the secondary ions with small angles to enter into the working-gas region. The TPC approach improves the spatial resolution of GEM neutron detectors through the time information, while the stoppinglayer method proposed in this work can be regarded as an improvement method in the perspective of space. Therefore, it can be also considered as a complementary approach in contrast with the TPC to improve the spatial resolution of the GEM neutron detector. In this paper, $1 \mu \mathrm{m}$-thick ${ }^{10} \mathrm{~B}_{4} \mathrm{C}$ is used as the neutron converter of the single GEM neutron detectors, and the titanium stopping layers in experimental studies have three specifications: $2 \mu \mathrm{m}, 2.5 \mu \mathrm{m}$, and $3 \mu \mathrm{m}$. A numerical analysis is executed for the influences of the stopping layer in the emitting angle of ion products. The Monte Carlo program FLUKA [2426]is utilized to simulate the spatial resolution of the detectors, and the testing experiments have been executed on the neutron beam line \#20 (BL20) at the CSNS. The influence of Ti-layer thickness to the spatial resolution is also presented.

\section{PRINCIPLES AND SIMULATIONS OF THE IMPROVEMENT FOR SPATIAL RESOLUTION}

\section{A. Principles and Simulations of the Improvement of Spatial Resolution}

The nuclear reaction of thermal neutrons with the isotope ${ }^{10} \mathrm{~B}$ is as follows [5]:

$\mathrm{n}+{ }^{10} B \rightarrow\left\{\begin{array}{lr}\alpha+{ }^{7} \mathrm{Li}+2.31 \mathrm{MeV}+\gamma(0.48 \mathrm{MeV}) & 94 \% \\ \alpha+{ }^{7} \mathrm{Li}+2.79 \mathrm{MeV} & 6 \%\end{array}\right.$,

The dominant reaction with the branching ratio of $94 \%$ releases a total kinetic energy of $2.31 \mathrm{MeV}$, and the $\alpha$-particles and ${ }^{7} \mathrm{Li}$ ions share the total energy. The two ions obey the law of conservation of momentum, so they will be scattered backto-back after the absorption reaction. The kinetic energy is respectively about $1.47 \mathrm{MeV}$ and $0.84 \mathrm{MeV}$ for the $\alpha$ particle and ${ }^{7} \mathrm{Li}$ ion for the branching ratio of $94 \%$. As a result, only one ion will emit into the working gas, and the other one will be scattered in an opposite direction and stop in the cathode.

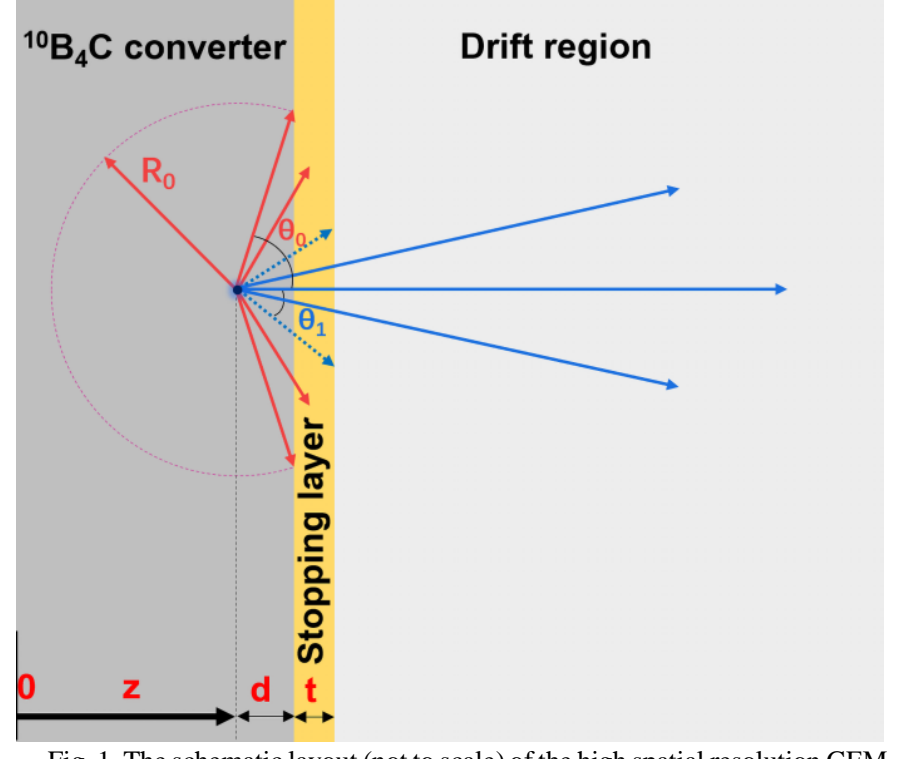

Fig. 1. The schematic layout (not to scale) of the high spatial resolution GEM neutron detector with a stopping layer.

The principle of spatial-resolution improvement for the GEM neutron detector is illustrated in Fig. 1. After the nuclear reaction of thermal neutron with the ${ }^{10} \mathrm{~B}$ nuclei, the ion products 
will be emitted isotropically. In the absence of the stopping layer and ignoring the track deflection, the maximum emitting angle $\left(\theta_{0}\right)$ of secondary ions relative to the surface normal is $\cos ^{-1}\left(d / R_{0}\right)$, where $d$ is the location of the ${ }^{10} \mathrm{~B}(\mathrm{n}, \alpha)^{7} \mathrm{Li}$ reaction relative to the ${ }^{10} \mathrm{~B}_{4} \mathrm{C}$ layer surface facing the working-gas region and $R_{0}$ the ion range in the ${ }^{10} \mathrm{~B}_{4} \mathrm{C}$. The value of $\theta_{0}$ can be near $90^{\circ}$ for a reaction location very close to the emitting surface. The ion range in the working gas can reach several millimeters, e.g., in argon at $1 \mathrm{~atm}$ pressure, it is $\sim 7.8 \mathrm{~mm}$ and $\sim 4.1 \mathrm{~mm}$ for the $1.47-\mathrm{MeV} \alpha$ particle and the $0.84-\mathrm{MeV}^{7} \mathrm{Li}$ ion, respectively [27]. Consequently, the transverse motion of ions entering into the working-gas region with large angles will play a dominant role in the resolution degradation for the GEM neutron detectors. In the case of coating a stopping layer on top of the ${ }^{10} \mathrm{~B}_{4} \mathrm{C}$ converter, the emitting angle will be narrowed down to a range of $\theta_{l}$ as shown in Fig. 1, because the extra stopping layer will exhaust the kinetic energy of ion products with relatively large angles.

The analytical or at least semi-analytical method to solve the maximum emitting angle of ion products could benefit to interpret the principles and give a comprehensive physical picture of the problem. In the following analytical process, only the reaction of $94 \%$ branching-ratio is considered in the relevant calculations. Assuming a secondary ion entering into the stopping layer with a kinetic energy of $E_{l}$, which is exactly sufficient to arrive at the stopping-layer surface. For the sake of simplicity, it is assumed that the ion tracks are along straight lines, i.e., the deflection of the tracks by the Coulomb scattering is ignored, so the isotropic emission of ions remains valid within the maximum polar angle for $\mathrm{B}_{4} \mathrm{C}$ converter with the stopping layer on top of it. The fundamental geometric relation is as follows:

$$
\left\{\begin{array}{l}
\cos \theta_{1}=t / l^{\prime}=(d+t) /\left(l+l^{\prime}\right) \\
l=R\left(E_{0}\right)-R\left(E_{1}\right) \\
l^{\prime}=R^{\prime}\left(E_{1}\right)
\end{array},\right.
$$

where $t$ is the thickness of the stopping layer, $R$ and $R^{\prime}$ are respectively the ion range in the medium of the converter and stopping layer as a function of ion energy, $l$ and $l$ ' are respectively the maximum track length (at the maximum emitting angle) of ions in the $\mathrm{B}_{4} \mathrm{C}$ converter and stopping layer, and $E_{0}$ is the initial energy of ion products at the nuclearreaction position. Therefore, it is essential to obtain the rangeenergy relations to solve (2). Fig. 2 shows the ion range versus the kinetic energy, which is provided by the SRIM program [27] The material of the stopping layer is set to be titanium. The initial energy of the ion products is taken as the maximum kinetic energy of the data points for investigation. For an ionenergy lower than several $\mathrm{MeV}$, the range doesn't follow the usual exponential function of kinetic energy. A semi-empirical formula $\left(a \cdot e^{b E}+c\right)$ is proposed to fit the range-energy curve within this energy range [28], and it can be seen the fitting agrees well with data points.
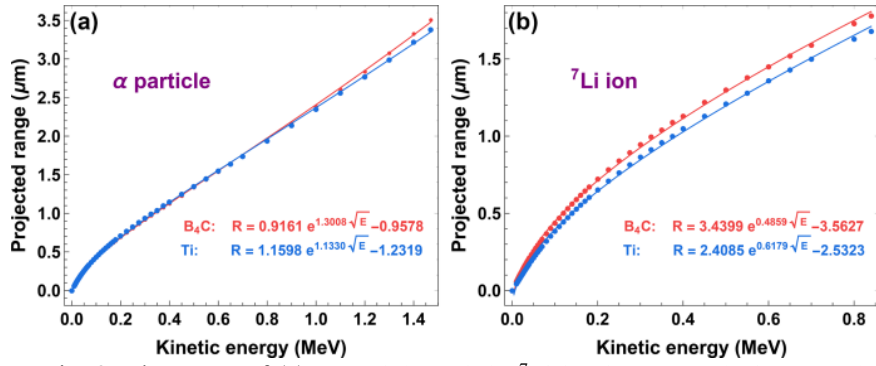

Fig. 2. The range of (a) $\alpha$-particle and (b) ${ }^{7} \mathrm{Li}$-ion in $\mathrm{B}_{4} \mathrm{C}$ and $\mathrm{Ti}$ versus the kinetic energy. Data is provided by the SRIM program.

Substituting the range formula into (2) and eliminating variables, we can obtain the following equation:

$d \cdot l^{\prime}=\left\{\begin{array}{l}t \cdot\left(4.4350-0.9161 \times e^{1.1481 \times \ln (1.0622+0.8622 l)}\right) \text { for } \alpha \\ t \cdot\left(5.3697-3.4399 \times e^{0.7864 \times \ln (1.0514+0.4152 l)}\right) \text { for }{ }^{7} \mathrm{Li}\end{array}\right.$.

Equation (3) is a transcendental equation, so it does not have an analytical solution, thus the numerical approach is necessary. Fig. 3 depicts the root distribution of track length $l^{\prime}$ in Ti stopping layer for given values of $d$ and $t$. The red lines marked with $d$ values represents the curve sets of function $d \cdot l^{\prime}$. Blue and orange lines marked with $t$ values respectively represent the curve sets of function values corresponding to the right hand of (3) for the $\alpha$ particle and $\mathrm{Li}$ ion, and these curves converge on a point on the horizontal axis where is respectively the range of two ion products in Ti. The intersection points of the two different curve sets represent the root of $l$ '. However, $l$ ' should be greater than the thickness of stopping layer $t$, otherwise, the intersection points represent the imaginary roots for the final calculation of the cosine of the angle from the perspective of mathematics. The real roots corresponding to different values of $d$ and $t$ are shown as the magenta point markers in the figure. For a same thick Ti layer, one can see a deeper reaction position with respect to the $\mathrm{B}_{4} \mathrm{C}$ surface facing to the Ti-layer have a smaller maximum of track length, i.e., a smaller maximum of emitting angle will be presented. For a same reaction position (i.e., $d$ ), the $l$ ' values won't increase proportionally with the increase of the Ti-layer thickness $t$, which implies a decrease of the maximum of emitting angle (calculated as $\cos ^{-1}\left(t / l^{\prime}\right)$ ) of ion products.

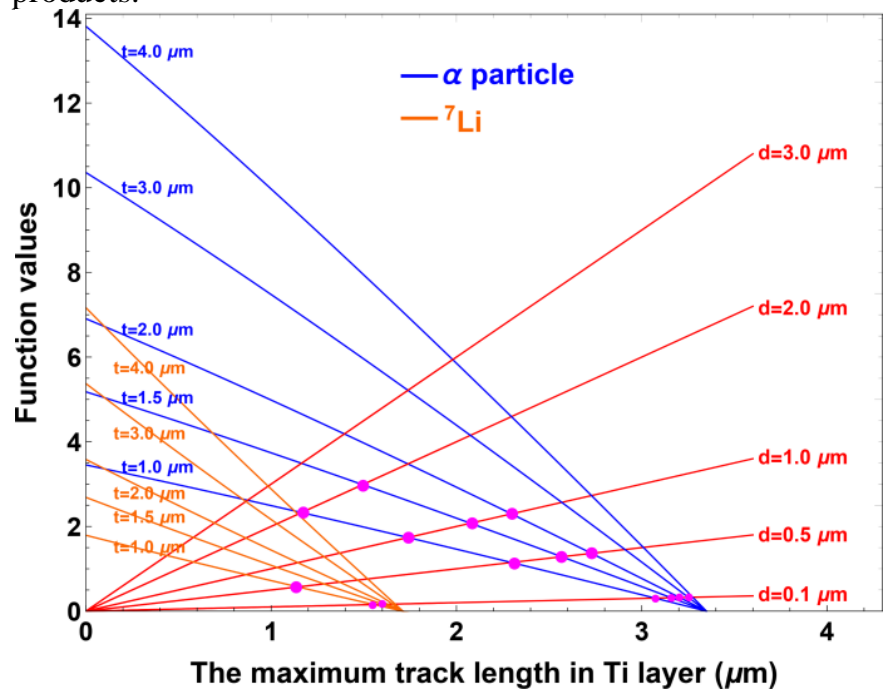

Fig. 3. The root distribution of the maximum track length $l$ ' in the Ti stopping layer for given values of $d$ and $t$. 

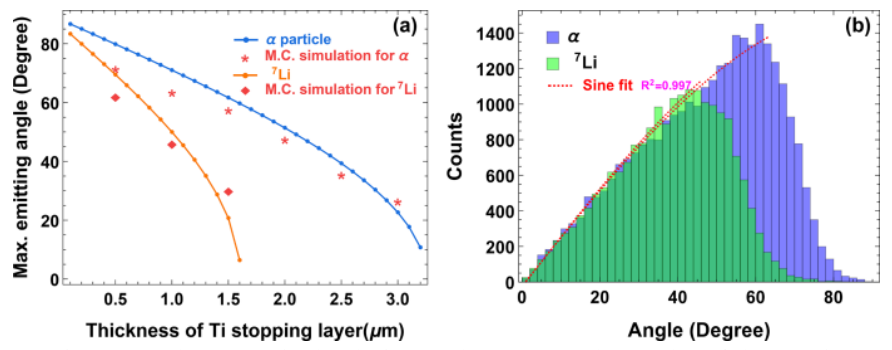

Fig. 4. (a)The numerical solution and Monte Carlo results of the maximum emitting angle of secondary ions entering into the working gas versus the thickness of the Ti stopping layer. The reaction point $d$ is set to be $0.1 \mu \mathrm{m}$. (b) The angular distribution of secondary ions performed by the FLUKA program for $d=0.1 \mu \mathrm{m}$ and $t=1 \mu \mathrm{m}$.

Fig. 4 presents numerical and Monte Carlo results of the maximum emitting angle versus the thickness of Ti stopping layer, which is for the case of nuclear reaction takes place at $d=0.1 \mu \mathrm{m}$. As presented in Fig. 4a, results of numerical approach approximately agree with that provided by the Monte Carlo program FLUKA. The numerical results indicate that the maximum emitting-angle decreases as increasing the thickness of the stopping layer, which decreases from $\sim 87^{\circ}(0.1-\mu \mathrm{m}$-thick Ti) to $\sim 23^{\circ}\left(3-\mu \mathrm{m}\right.$-thick Ti) for $\alpha$ particles and from $\sim 83^{\circ}(0.1$ $\mu \mathrm{m}$-thick Ti) to $\sim 21^{\circ}(1.5-\mu \mathrm{m}$-thick $\mathrm{Ti})$ for ${ }^{7} \mathrm{Li}$ ions. Fig. $4 \mathrm{~b}$ illustrates the way to determine the maximum emitting angle from the results of the Monte Carlo program. Due to the Coulomb scattering, the angular distribution of ions will have a tail for angle beyond the peak point. However, the Coulomb scattering is not considered in the numerical approach, so the peak point of angular distribution is selected as the maximum emitting angle to validate the numerical analysis. It is known that an isotropic emission of products in the nuclear reaction of thermal neutrons will have a $\sim \sin \theta$ distribution in the polar angle $\theta$. As the FLUKA simulations shown in Fig. 4b, the angular distribution is still an approximate sinusoidal one for angles lower than the peak point. Therefore, for comparison with the numerical method, it is reasonable to select the peak point as the maximum angle within which an isotropic emission is still roughly valid.

\section{B. Monte Carlo simulations of the spatial resolution.}

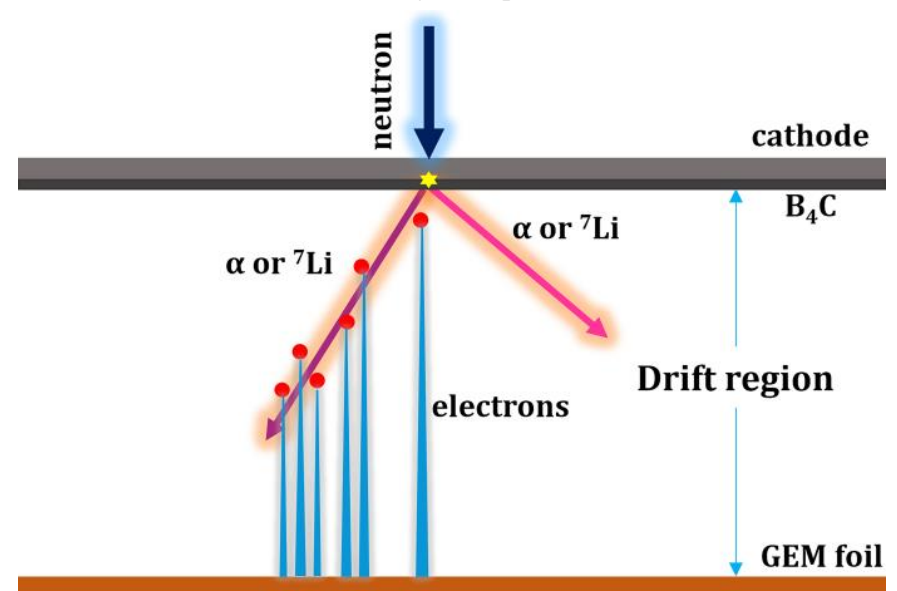

Fig. 5. The calculation model for the spatial resolution.

Simulations for the spatial resolution are also performed by FLUKA. The calculation model is shown in Fig. 5. To approximately treat and simplify the simulations, the electron multiplication and the collection stages are not considered, i.e., only the neutron reactions occurring in the converter and the ionization process in the drift region are involved. Assuming an operation voltage of $1600 \mathrm{~V}$ for our detectors, the electric field strength is about $2900 \mathrm{~V} / \mathrm{cm}$ in the induction region according to the resistor of the high-voltage chain (see section III). The transverse diffusion coefficient for gas mixture $\mathrm{Ar} / \mathrm{CO}_{2}$ (90\%:10\%) is about $360 \mu \mathrm{m} / \sqrt{\mathrm{cm}}$ [29], thus the transverse diffusion in the induction region of a $2-\mathrm{mm}$ gap is about 0.16 $\mathrm{mm}$, therefore, for an approximate treatment, it is reasonable to neglect the electron transverse diffusion in the induction region and employ the above assumptions.

Fig. 6 shows the simulated ion tracks in the drift region for different cathode configurations for $0.025-\mathrm{eV}$ neutrons perpendicularly incident on the cathodes. The drift gap in simulations is assumed to be $4 \mathrm{~mm}$. The coordinate of the incident point is set at $(0,0,0)$, and the thickness of the Ti layer varies from 0 to $3 \mu \mathrm{m}$. As can be seen, a narrower angle distribution is presented for a thicker Ti layer. Furthermore, the ${ }^{7} \mathrm{Li}$-ion tracks are vanished in the drift region when the stopping-layer thickness is greater than $2 \mu \mathrm{m}$, i.e., only $\alpha$ particles contribute to the final signal for Ti layer thicker than 2 $\mu \mathrm{m}$ (more precisely, $1.7 \mu \mathrm{m}$, the range of $0.84-\mathrm{MeV}^{7} \mathrm{Li}$ in $\mathrm{Ti}$ ). From the simulated tracks, an obvious improvement of the spatial resolution could be imagined.
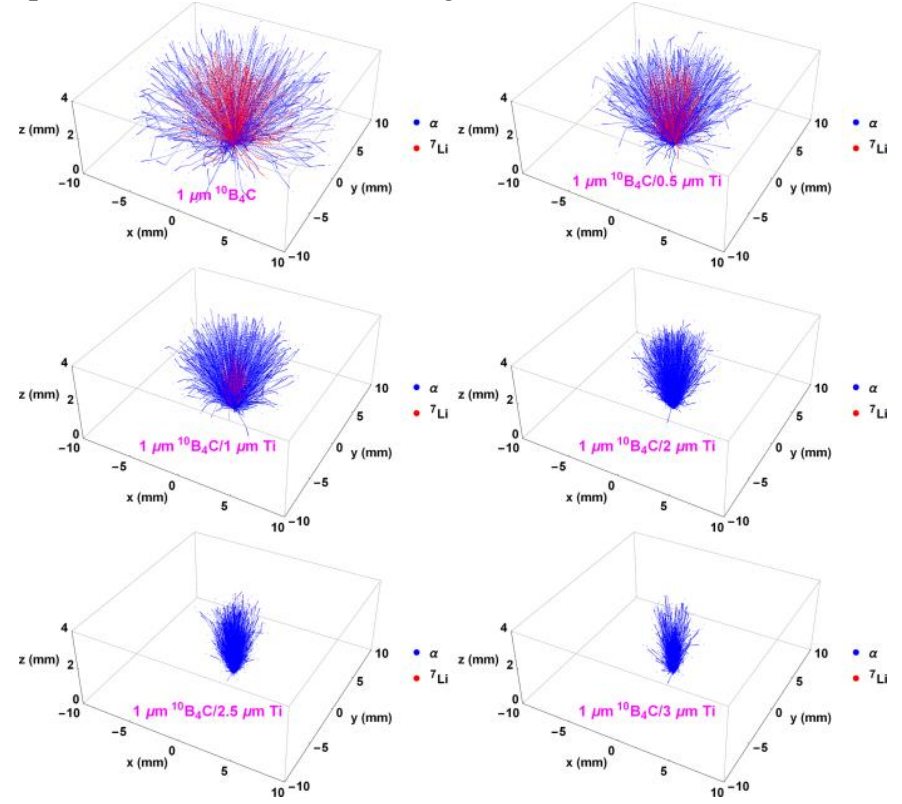

Fig. 6. The simulated ion tracks in the drift region for different cathode configurations. The energy of perpendicularly incident neutrons is $0.025 \mathrm{eV}$, and the neutrons incident point is set at $(0,0,0)$.

The main physical quantity involved in simulations of spatial resolution is the energy deposition of secondary ions in the working-gas of drift region. The coordinates of ionization events together with the values of energy deposition will be extracted to obtain the resolution. In principle, a point-like source can be set to obtain the point spread function (PSF) of the detector system, then the PSF can be deduced to the spatial resolution [30]. Generally, a strong neutron-absorption material (e.g., $\mathrm{Cd}$ or aluminum-boron-alloy) with a rather small hole is needed to collimate the neutron beam into an approximately ideal point source. However, even though suppose a very small 
hole is a good approximation for a dimensionless point, a rather long test time is necessary to achieve adequate statistics, which is not a cost-effective method in the experiments utilizing neutron beam. Experimentally, the line spread function (LSF) or edge spread function (ESF) is more commonly used to determine the spatial resolution [30]. This paper employs the LSF method, additionally, as depicted in section IV, the slanted-angle slit method is adopted to get the presampled LSF to overcome the limitation of the pitch size of the readout board and avoid aliasing, which can get much better sampling than the readout pitch. For convenient comparison, the slanted-angle slit method is also performed in the Monte Carlo simulations to obtain the LSF and the following spatial resolution of the GEM neutron detector. The algorithm for solving the spatial resolution is based on the method proposed by Fujita [31].
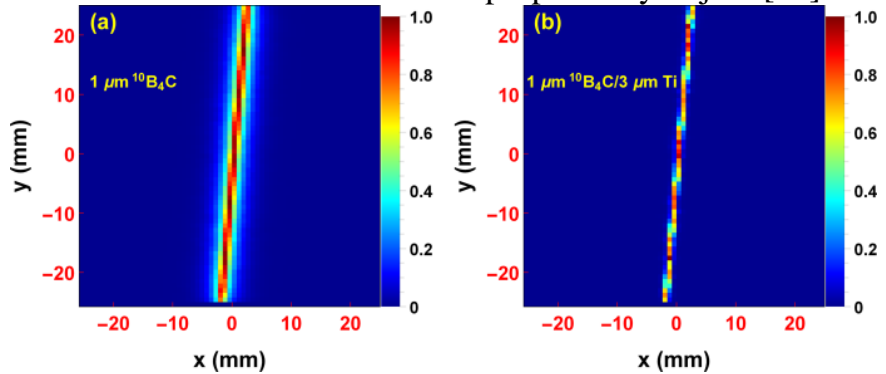

Fig. 7. The reconstructed $5^{\circ}$ slanted-slit image of ideal $0.025-\mathrm{eV}$ neutron line source based on the energy deposition for the cathode with (a) $1 \mu \mathrm{m}{ }^{10} \mathrm{~B}_{4} \mathrm{C}$ and (b) $1 \mu \mathrm{m}{ }^{10} \mathrm{~B}_{4} \mathrm{C} / 3 \mu \mathrm{m} \mathrm{Ti}$.

An ideal $0.025-\mathrm{eV}$ neutron line source with a $5^{\circ}$ slanted angle relative to the $y$-axis is defined in FLUKA. The reconstructed slit images are shown in Fig. 7, and the bin width for the 2D reconstruction is $0.78 \mathrm{~mm}$ that is the same with the actual strip pitch of our GEM neutron detector (see section III). Compared with the diffuse halo for the cathode coated with $1 \mu \mathrm{m}{ }^{10} \mathrm{~B}_{4} \mathrm{C}$, one can see the halo around the slit almost vanishes for cathode coated with $1 \mu \mathrm{m}{ }^{10} \mathrm{~B}_{4} \mathrm{C} / 3 \mu \mathrm{m} \mathrm{Ti}$, which implies an obvious improvement of the spatial resolution for the latter one. Fig. 8 shows the simulated distribution and the Gaussian fit for detectors with different thick Ti stopping layer. As can be seen, the FWHM spatial resolution value reduces gradually from 1.77 $\mathrm{mm}\left(1 \mu \mathrm{m}{ }^{10} \mathrm{~B}_{4} \mathrm{C}\right)$ to $0.81 \mathrm{~mm}\left(1 \mu \mathrm{m}{ }^{10} \mathrm{~B}_{4} \mathrm{C} / 3 \mu \mathrm{m} \mathrm{Ti}\right)$ as increasing the thickness of the $\mathrm{Ti}$ stopping layer.

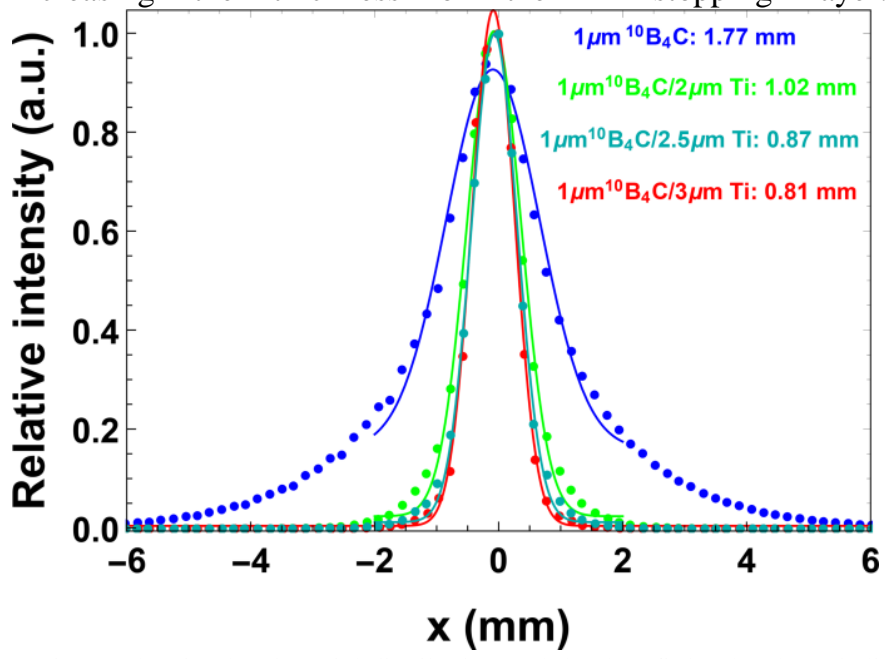

Fig. 8. The simulated spatial distribution and the data fit by the slanted-slit method for the detector cathode with different configurations.

\section{DETECTOR CONFIGURATIONS}

The schematic of the GEM neutron detector, the detector photograph, and the readout board are shown in Fig. 9. And the basic design parameters are listed in Table I. The detector consists of the gas chamber filled with the gas mixture of $\mathrm{Ar} / \mathrm{CO}_{2}$ (90\%:10\% by volume) and the readout electronics, while the electronics is separated from the gas chamber for adding an electromagnetic shield.

Table I. The basic design parameters of the GEM neutron detector.

\begin{tabular}{lc}
\hline Design parameters & VALUE \\
\hline Overall size & $253 \mathrm{~mm} \times 210 \mathrm{~mm} \times 37.5 \mathrm{~mm}$ \\
Sensitive area & $50 \mathrm{~mm} \times 50 \mathrm{~mm}$ \\
Total readout channels & 128 \\
Pitch of readout strips & $0.78 \mathrm{~mm}$ \\
Filled gas & $90 \% \mathrm{Ar}+10 \% \mathrm{CO}_{2}$ by volume \\
Gas pressure & $1 \mathrm{~atm}$ \\
Standard bias voltage & $1600 \mathrm{~V}$ \\
Gaps of drift/induction region & $4 \mathrm{~mm} / 2 \mathrm{~mm}$ \\
Chamber material & Aluminum \\
\hline \hline
\end{tabular}

The GEM neutron detector is operated at a gas pressure of 1 atm and a voltage of $1600 \mathrm{~V}$. A high-voltage chain is used to distribute the voltage on the cathode, the upper and lower surface of the GEM foil, respectively. The resistor of the high-voltage chain from top to bottom is respectively $8.3,4.7$, and $10 \mathrm{M} \Omega$. The gap of the drift region is $4 \mathrm{~mm}$, and that of the induction region is $2 \mathrm{~mm}$. The active area of the detector is $50 \mathrm{~mm} \times 50 \mathrm{~mm}$.

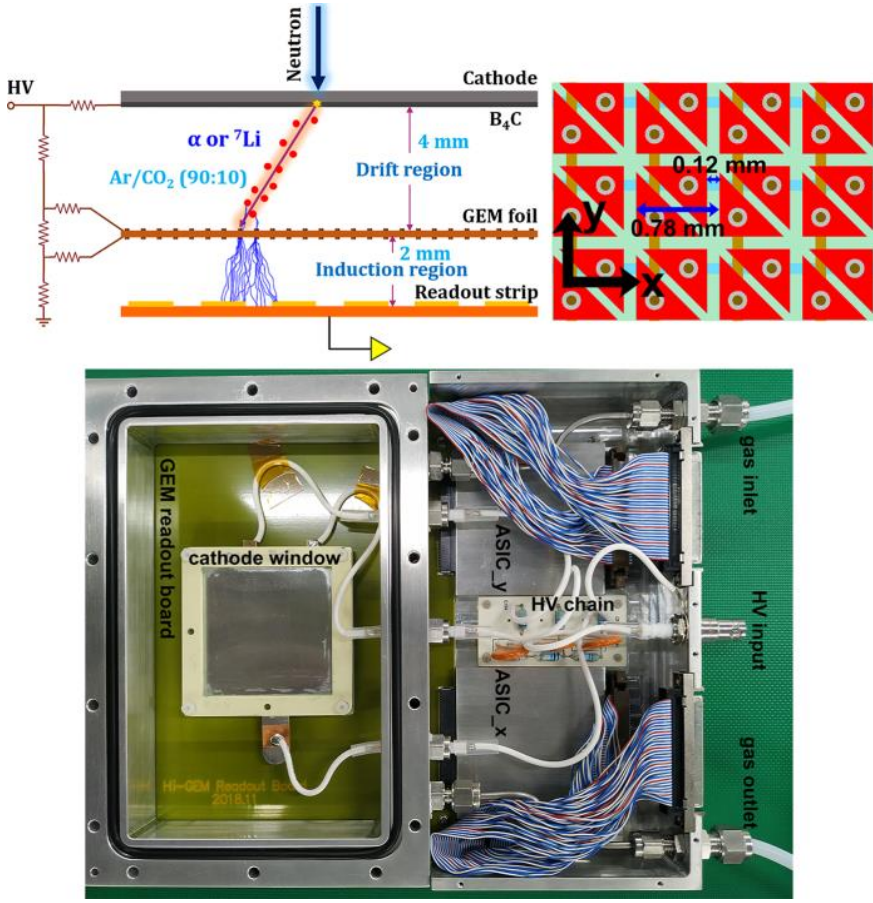

Fig. 9. The GEM neutron detector schematic (top left), its readout board (top right), and the photograph of the GEM neutron detector (bottom).

The cathode is a $0.1-\mathrm{mm}$-thick aluminum plate coated with a 1 - $\mu$ m-thick $\quad{ }^{10} \mathrm{~B}_{4} \mathrm{C} \quad\left(96 \%\right.$-enriched $\left.\quad{ }^{10} \mathrm{~B}\right)$ converter layer with/without a Ti stopping layer coated on top of the ${ }^{10} \mathrm{~B}_{4} \mathrm{C}$ layer. The thickness of the Ti layer adopted in experiments is $2 \mu \mathrm{m}, 2.5$ $\mu \mathrm{m}$, and $3 \mu \mathrm{m}$, respectively. For purpose of comparison, the cathode converter without Ti layer is also prepared. The readout board is based on the PCB technology, and there are 64-channel strips in each dimension with a strip pitch of $0.78 \mathrm{~mm}$. The ceramic GEM foil is also manufactured by the PCB technology. 
The $200-\mu \mathrm{m}$ diameter holes are drilled through a copper-coated $200-\mu \mathrm{m}$ thick ceramic board. The hole pitch is $600 \mu \mathrm{m}$, and the etched rim has a width of $80 \mu \mathrm{m}$. The readout electronics system is based on an analogue frontend readout chips (CIPix ASIC chip developed by the Heidelberg ASIC laboratory) in combination with a field-programmable gate array (FPGA) data processing unit [12]. The CIPix chip integrates 64 independent analogue channels comprising a charge-sensitive preamplifier, a shaper, and a discriminator, then the output signals are digitized by an AC-coupled comparator with configurable polarity sampled at a clock rate of $10 \mathrm{MHz}$. The two-dimensional position information of the incident neutron is processed with a real-time event reconstruction algorithm in the FPGA by coincidence correlation This reconstruction algorithm evaluates the data from the readout structure together with the time stamp information $t$ synchronized by an external $\mathrm{T} 0$ signal to one neutron event $(x, y, t)$.

\section{EXPERIMENTAL RESULTS AND DISCUSSIONS}

The test experiments of the GEM neutron detector were carried out at the BL20 of CSNS. The BL20 is a test beam line with a decoupled \& poisoned hydrogen moderator and a beam chopper. The neutron flux is about $10^{6} /\left(\mathrm{cm}^{2} \cdot \mathrm{s}\right)$. Fig. 10 depicts the experimental setup at the BL20. The neutron beam exit port is rectangular in shape with a size of $36 \mathrm{~mm} \times 40 \mathrm{~mm}$, and a 5$\mathrm{cm}$-thick beam limiter with a hole of $20 \mathrm{~mm}$ in diameter is placed in front of the GEM detector.

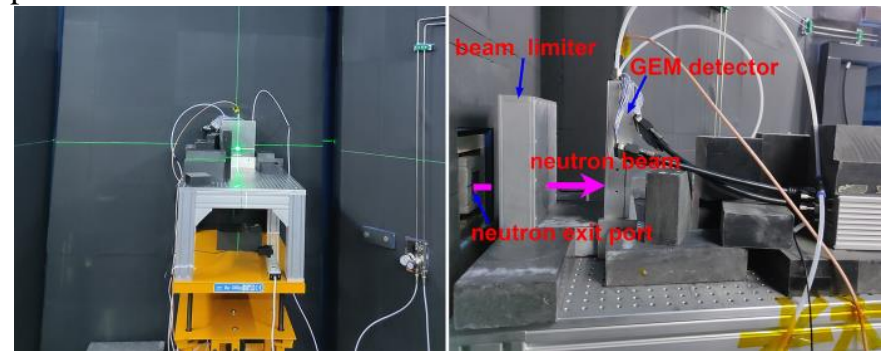

Fig. 10. Photographs of the experimental setup at the BL20 of CSNS. The left photograph depicts the alignment of the detector test system, and the right one shows the detailed setup.

The flight distance between the moderator and the beam exit port is $11.4 \mathrm{~m}$, and the distance between the exit port with the detector is $23 \mathrm{~cm}$. Based on the De Broglie formula, the neutron wavelength spectrum can be obtained through a conversion from the neutron time of flight (TOF) obtained by the GEM detector to the neutron wavelength:

$$
\lambda \simeq \frac{0.0039615 t}{d_{f}} \quad[\AA],
$$

where $\lambda$ is the neutron wavelength in units of $\AA, t$ the neutron TOF ( $\mu \mathrm{s})$, and $d_{f}$ the flight distance (m). Fig 11. shows the neutron beam spot imaging and the neutron wavelength spectrum acquired by the GEM detector with the 1- $\mu \mathrm{m}$-thick ${ }^{10} \mathrm{~B} 4 \mathrm{C}$ converter. During the test, the neutron beam chopper keeps running. Fig. 11a displays a clear spot boundary and a circular beam spot with a diameter size of $\sim 20 \mathrm{~mm}$, which is consistent with the hole size of the beam limiter. The neutron wavelength shown in Fig. $11 \mathrm{~b}$ ranges from about 1 to $5.5 \AA$, and the peak is around $2.5 \AA$. The apparent valley points on the spectrum curve correspond to the reference values of aluminum main Bragg edges [32], which are originated from the neutron interaction with the detector shell, more precisely, the $0.5-\mathrm{mm}$ thick aluminum window. The detected positions of Bragg edges are almost the same as the standard ones, which demonstrates the correctness and the high precision of the TOF measurement.
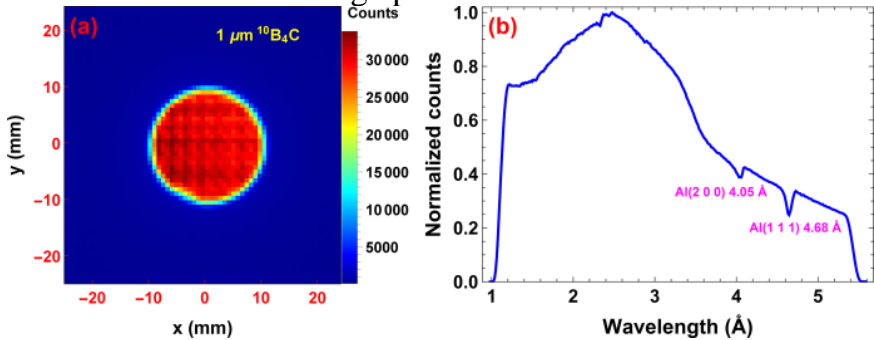

Fig. 11. (a) The neutron beam spot, and (b) the wavelength spectrum measured by the GEM detector with a converter of $1-\mu \mathrm{m}$-thick ${ }^{10} \mathrm{~B}_{4} \mathrm{C}$ at the BL20. The text labels at the apparent valley points in (b) correspond to the reference positions of the aluminum main Bragg edges.

The spatial resolution is measured with a $0.1-\mathrm{mm}$-wide slit mask made of cadmium. In measurements of the spatial resolution, the Cd-made slit mask is mounted at the entrance window of the GEM neutron detector. Fig. 12 shows the slit profile obtained by the detectors and the projection of the profile on the $x$-axis. As shown in Fig. 12b, one can note that only three strips have responses to the collimated neutron beam behind the slit mask. Although a Gaussian fitting can be employed to extract the relevant parameters, however, there may exist the so-called aliasing effects caused by the coarsesampling [30].
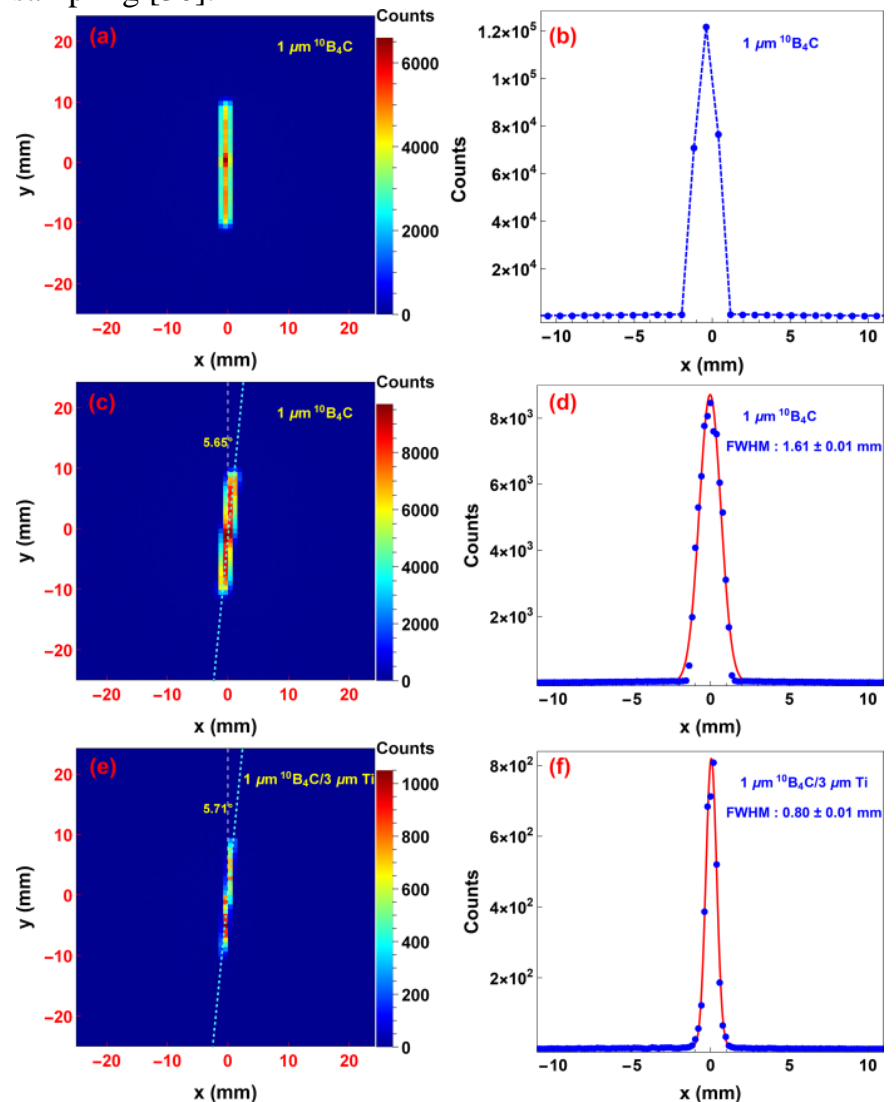

Fig. 12. The slit imaging obtained by the GEM neutron detector with cathode configurations of $1 \mu \mathrm{m}{ }^{10} \mathrm{~B}_{4} \mathrm{C}$ and $1 \mu \mathrm{m}{ }^{10} \mathrm{~B}_{4} \mathrm{C} / 3 \mu \mathrm{m} \mathrm{Ti}$, and the $1 \mathrm{D}$ distribution through the projection on the $x$-axis. (a) the imaging of vertical slit and (b) the corresponding 1D distribution; (c) the imaging of $5^{\circ}$ slanted slit and (d) the corresponding 1D distribution for the detector with $1 \mu \mathrm{m}{ }^{10} \mathrm{~B}_{4} \mathrm{C}$ configuration; (e) the imaging of $5^{\circ}$ slanted slit and (f) the corresponding $1 \mathrm{D}$ distribution for 
the detector with $1 \mu \mathrm{m}{ }^{10} \mathrm{~B}_{4} \mathrm{C} / 3 \mu \mathrm{m}$ Ti configuration

To improve the precision in the measurement procedure of the spatial resolution, the slanted-slit method is adopted. The LSF could be synthesized into the presampled LSF by taking the imaging profiles at different vertical locations, and thus the sampling with sub-pixel spacing intervals can be accomplished. In our experiment, the slanted angle of the slit is $5^{\circ}$ relative to the vertical direction of the readout board, and the slit width is also $0.1 \mathrm{~mm}$. Fig. 12 shows the imaging of a vertical slit, $5^{\circ}$ slanted slit, and the corresponding 1D distribution along the $x$ axis and the fit for them. Fig. 12c and Fig. 12e show the fitting angle is respectively about $5.65^{\circ}$ and $5.71^{\circ}$, which is well consistent with the design angle. The small divergence is brought by the machining error, the mounting error of the slit mask, or the positioning error of the GEM detector. Fig. 12d and Fig. 12f show a much better sampling, and the spatial resolution is respectively about $1.61 \mathrm{~mm}$ and $0.80 \mathrm{~mm}$ for the 1 $\mu \mathrm{m}$ thick ${ }^{10} \mathrm{~B}_{4} \mathrm{C}$ and $1 \mu \mathrm{m}{ }^{10} \mathrm{~B}_{4} \mathrm{C} / 3 \mu \mathrm{m}$ Ti configured detector.

Fig. 13 shows the experimental results of the spatial resolution compared with the simulations as increasing the thickness of the $\mathrm{Ti}$ stopping layer. Furthermore, simulated results corrected by the neutron spectrum of the BL20 are also presented. On the whole, the experimental results basically agree with the simulations. Both present a declining tendency for the value of spatial resolution as the thickness of the Ti layer increases. As can be seen, the spatial resolution approaches the sub-mm level when the Ti-layer thickness is $2 \mu \mathrm{m}$, and it is $\sim 0.8$ $\mathrm{mm}$ when the thickness is reached to $3 \mu \mathrm{m}$. The maximum relative difference of the spatial resolution between the experimental and simulated value is $\sim 9.6 \%$ for the detector of 1 $\mu \mathrm{m}{ }^{10} \mathrm{~B}_{4} \mathrm{C}$. However, it can be concluded from the test experiments and simulations that a 3 - $\mu \mathrm{m}$-thick Ti stopping layer could improve the spatial resolution of the $\mathrm{B}_{4} \mathrm{C}$-based GEM neutron detector to a steady sub-mm region.

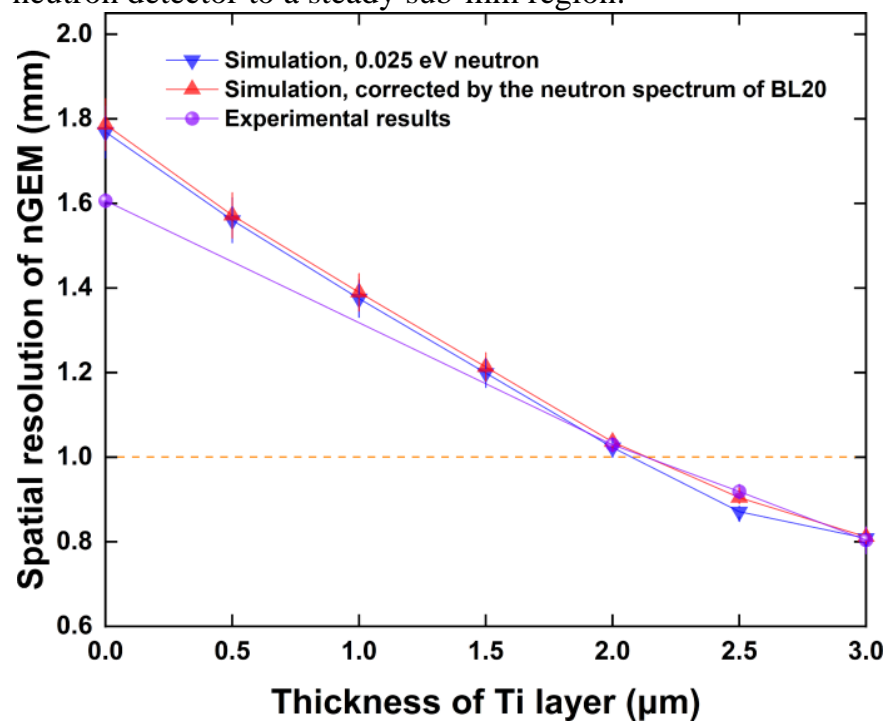

Fig. 13 The experimental and simulated spatial resolution versus the thickness of the Ti layer.

The Cd mask of the letter " $E$ " is also utilized to observe the imaging performances of the GEM neutron detector. Fig. 14 presents the experimental results for cathode configurations with $1 \mu \mathrm{m}{ }^{10} \mathrm{~B}_{4} \mathrm{C}$ converter and $1 \mu \mathrm{m}{ }^{10} \mathrm{~B}_{4} \mathrm{C}$ converter with different thick Ti stopping layers. The Cd mask of the letter "E" has a size of $1 \mathrm{~cm} \times 1 \mathrm{~cm}$ (see the inlay photograph in Fig. 14a). And slits of the hollow part are $2 \mathrm{~mm}$ wide in both directions, across which the neutrons are allowed to hit on the detector. Compared with the $1 \mu \mathrm{m}-{ }^{10} \mathrm{~B}_{4} \mathrm{C}$ case, the detector with Ti layer apparently reveals a sharper edge and more uniform imaging in the hollow parts. The improvement effect is more apparent for a thicker Ti layer, in other words, a higher spatial resolution is achieved.
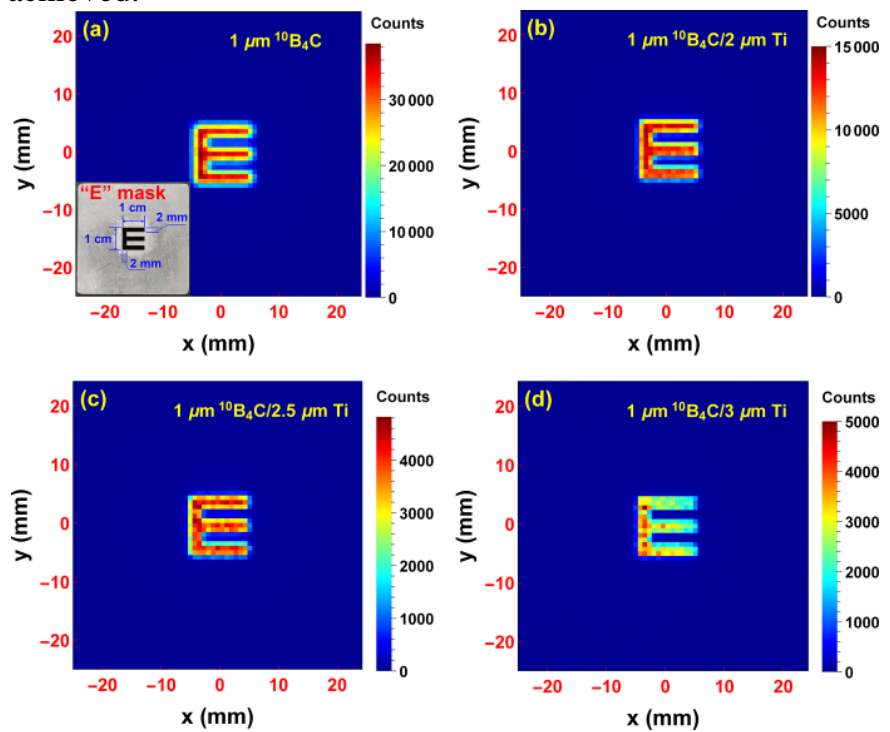

Fig. 14. The neutron imaging of a Cd-made letter "E" mask using the GEM detector with different cathode configurations at the BL20 of CSNS: (a) $1 \mu \mathrm{m}$ ${ }^{10} \mathrm{~B}_{4} \mathrm{C}$, (b) $1 \mu \mathrm{m}{ }^{10} \mathrm{~B}_{4} \mathrm{C} / 2 \mu \mathrm{m} \mathrm{Ti}$, (c) $1 \mu \mathrm{m}{ }^{10} \mathrm{~B}_{4} \mathrm{C} / 2.5 \mu \mathrm{m} \mathrm{Ti}$, (d) $1 \mu \mathrm{m}{ }^{10} \mathrm{~B}_{4} \mathrm{C} / 3 \mu \mathrm{m}$ Ti. The inlay of (a) shows the size of the Cd-made letter "E" mask.

\section{CONCLUSION}

Spatial resolution is an important parameter of the GEM detector for its utilization in the neutron detection. A submillimeter resolution will be required in some newly built neutron instruments at the CSNS. This research proposes a new approach of adding a stopping layer on top of the solid neutron converter ${ }^{10} \mathrm{~B} 4 \mathrm{C}$ to improve the spatial resolution of the GEM neutron detector. Monte Carlo simulations point out that the spatial resolution can be improved to the sub-millimeter level by adding a 3- $\mu$ m-thick Ti layer on top of the ${ }^{10} \mathrm{~B}_{4} \mathrm{C}$ converter for detectors operated at 1 atm. Simulations of ion tracks indicate that the improvement is brought about by the restriction of the emission of ion products with large angles. The beam experiments executed at the BL20 of CSNS nicely demonstrate that this method is effective to improve the spatial resolution to a sub-millimeter level. Experimental results accord basically with simulations. The experimental spatial resolution is $\sim 1.61$ $\mathrm{mm}$ for the detector with $1-\mu \mathrm{m}$-thick ${ }^{10} \mathrm{~B}_{4} \mathrm{C}$ converter without $\mathrm{Ti}$ stopping layer, which is improved to $\sim 0.80 \mathrm{~mm}$ for the detector with $1-\mu \mathrm{m}$-thick ${ }^{10} \mathrm{~B}_{4} \mathrm{C}$ converter coating with a $3-\mu \mathrm{m}$ thick Ti stopping layer. This study is useful for the applications of GEM neutron detectors under the circumstance of high spatial resolution and high-flux requirements. 


\section{REFERENCES}

[1] J. Wei, H. Chen, Y. Chen, Y. Chen, Y. Chi, C. Deng et al., "China Spallation Neutron Source: Design, R\&D, and outlook," Nucl. Instrum. Methods Phys. Res., Sect. A, vol. 600, pp. 10-13, 2009.

[2] A. J. Dianoux, and G. Lander, "Neutron Data Booklet," 2nd edition, Philadelphia, PA, USA: OCP Science, 2003, pp. 167-171.

[3] D. A. Shea, and D. Morgan, "The Helium-3 Shortage: Supply, Demand, and Options for Congress," Congressional Research Service, R41419, 2010

[4] R. T. Kouzes, A. T. Lintereur, and E. R. Siciliano, "Progress in alternative neutron detection to address the helium-3 shortage," Nucl. Instrum. Methods Phys. Res. Sect. A, vol. 784, pp. 172-175, 2015.

[5] G. F. Knoll, "Radiation Detection and Measurement," 4th Edition, New York, USA: WILEY John Wiley \& Sons, Inc, 2010, pp. 521-522.

[6] F. Sauli, "GEM: A new concept for electron amplification in gas detectors," Nucl. Instrum. Methods Phys. Res. Sect. A, vol. 386, pp. 531-534, 1997.

[7] F. Sauli, "The gas electron multiplier (GEM): Operating principles and applications," Nucl. Instrum. Methods Phys. Res. A, vol. 805, pp. 2-24 2016.

[8] M. Klein, H. Abele, D. Fiolka, and C. J. Schmidt, "CASCADE: A new efficient and position sensitive detector for thermal neutrons on large areas," AIP Conference Proceedings, vol. 596, pp. 193-198, 2001

[9] S. H. Park, Y. K. Kim, and J. K. Kim, "Neutron Detection with a GEM," IEEE Trans. Nucl. Sci. , vol. 52 pp. 1689-1692, 2005.

[10] S. Uno, M. Sekimoto, T. Murakami, M. Tanaka, S. Nakagawa, E. Nakano et al., "Development of Neutron Gaseous Detector with GEM," 2007 IEEE Nuclear Science Symposium Conference Record, pp. 4623-4626, 2007.

[11] T. Uchida, Y. Fujita, M. Tanaka, and S. Uno, "Prototype of a Compact Imaging System for GEM Detectors," IEEE Trans. Nucl. Sci. , vol. 55, pp. 2698-2703, 2008

[12] M. Klein, and C. J. Schmidt, "CASCADE, neutron detectors for highest count rates in combination with ASIC/FPGA based readout electronics," Nucl. Instrum. Methods Phys. Res. A, vol. 628, pp. 9$18,2011$.

[13] H. Ohshita, T. Otomo, S. Uno, K. Ikeda, T. Uchida, N. Kaneko et al., "Stability of neutron beam monitor for High Intensity Total Diffractometer at J-PARC," Nucl. Instrum. Methods Phys. Res. Sect. $A$, , vol. 672, pp. 75-81, 2012.

[14] M. Cortesi, R. Zboray, A. Kaestner, and H.-M. Prasser, "Development of a cold-neutron imaging detector based on thick gaseous electron multiplier," Rev. Sci. Instrum. , vol. 84, pp. 023305, 2013.

[15] M. Köhli, F. Allmendinger, W. W. Häußler, T. Schröder, M. Klein, M. Meven, and U. Schmidt, "Efficiency and spatial resolution of the CASCADE thermal neutron detector," Nucl. Instrum. Methods Phys. Res. A, vol. 828, pp. 242-249, 2016.

[16] G. Croci, A. Muraro, E. P. Cippo, G. Grosso, C. Höglund, R. H Wilton et al., "I-BAND-GEM: a new way for improving BANDGEM efficiency to thermal and cold neutrons," Eur. Phys. J. Plus, vol. 134, pp. 166-173, 2019.

[17] J. Zhou, Q. Xiu, X. Zhou, J. Zhou, L. Ma, C. J. Schmidt et al., "Highly efficient GEM-based neutron detector for China Spallation Neutron Source," Nucl. Instrum. Methods Phys. Res. A., vol. 953, pp. $163051,2020$.

[18] J. Zhou, X. Zhou, J. Zhou, H. Teng, J. Yang, Y. Ma et al., “A ceramic GEM-based neutron beam monitor for China Spallation NeutronSource," Nucl. Instrum. Methods Phys. Res. A, vol. 962, pp. 163593, 2020.

[19] J. Zhou, X. Zhou, J. Zhou, X. Jiang, J. Yang, L. Zhu et al., "A novel ceramic GEM used for neutron detection," Nucl. Eng. Technol., vol. 52, pp. 1277-1281, 2020.

[20] D. Pfeiffer, F. Resnati, J. Birch, R. H. Wilton, C. Höglund, L. Hultman et al., "The $\mu$ TPC Method: Improving the Position Resolution of Neutron Detectors Based on MPGDs," JINST 10 P04004, 2015.

[21] B. Flierl, R. Hertenberger, O. Biebel, and K. Zeitelhack, "TPC-like readout for thermal neutron detection using a GEM-detector," Nucl. Instrum. Methods Phys. Res. A., vol. 824, pp. 528-531, 2016.
D. Pfeiffer, F. Resnati, J. Birch, M. Etxegarai, R. Hallwilton, C. Höglund et al., "First measurements with new high-resolution gadolinium-GEM neutron detectors," JINST 11 P05011, 2016.

[23] Y. Zhang, H. Wu, J. Zhou, S. Zhao, and B. Hu, "Track identification and reconstruction in fast neutron detection by MPGD," Nucl. Instrum. Methods Phys. Res. A, vol. 906, pp. 68-76, 2018.

[24] A. Ferrari, P. Sala, A. Fassò, and J. Ranft, "FLUKA: A multi-particle transport code," Technical Report No. CERN-2005-10, INFN/TC_05/11, SLAC-R-773, CERN/INFN 2005, 2005.

[25] T. T. Böhlen, F. Cerutti, M. P. W. Chin, A. Fasso, A. Ferrari, P. G. Ortega et al., "The FLUKA Code: Developments and Challenges for High Energy and Medical Applications," Nucl. Data Sheets, vol. 120, pp. 211-214, 2014.

[26] G. Battistoni, T. Boehlen, F. Cerutti, P. W. Chin, L. S. Esposito, F. Alberto et al., "Overview of the FLUKA code," Annals of Nuclear Energy, vol. 82, pp. 10-18, 2015.

[27] J. F. Ziegler, and J. P. Biersack, SRIM-2013, http://www.srim.org, 2013.

[28] L. Tsoulfanidis, and S. Landsberger, "Measurement \& Detection of Radiation," 4th edition, Boca Raton, FL, USA: CRC Press, 2015, pp. 123.

[29] F. Sauli, "Gaseous Radiation Detectors," New York, USA: Cambridge University Press Cambridge, England, 2014, pp. 127.

[30] J. T. Bushberg, J. A. Seibert, E. M. Leidholdt, and J. M. Boone, "The Essential Physics of Medical Imaging," 3rd edition, Philadelphia, USA: Lippincott Williams \& Wilkins Press, 2011, pp. 61-64.

[31] H. Fujita, D. Y. Tsai, T. Itoh, K. Doi, J. Morishita, K. Ueda et al., "A simple method for determining the modulation transfer function in digital radiography," IEEE Trans. Med. Imaging, vol. 11, pp. 34-39, 1992.

[32] M. Harada, M. Teshigawara, M. Ohi, E.Klinkby, L. Zanini, K. Batkov et al., "Experimental validation of the brightness distribution on the surfaces of coupled and decoupled moderators composed of $99.8 \%$ parahydrogen at the J-PARC pulsed spallation neutron source," Nucl. Instrum. Methods Phys. Res. Sect. A, vol. 903, pp. $38-45,2018$. 